\title{
Infección por Clostridium difficile y el papel del trasplante de microbiota fecal
}

\section{Clostridium difficile infection and the role of the fecal microbiota transplant}

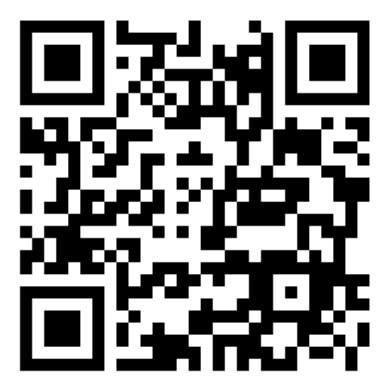

Recibido $21 / 01 / 2021$
${ }^{1}$ Dr. Mario Andrés Chacón Segura Investigador independiente, Alajuela, Costa Rica

(iD) https://orcid.org/0000-0003-0859-2517

${ }^{2}$ Dra. Laura Vanessa Bermúdez Durán Investigadora independiente, Limón, Costa Rica

(iD) https://orcid.org/0000-0002-4392-4794

${ }^{3}$ Dra. Diana Melissa Rojas Sancho Investigadora independiente, Heredia, Costa Rica

(iD) https://orcid.org/0000-0001-8339-3134

Corregido 26/04/2021
Aceptado 20/05/2021

\section{RESUMEN}

El Clostridium difficile es una bacteria anaeróbica, gram positiva capaz de colonizar el colon y producir diarrea aguda. Es una de las infecciones nosocomiales más importantes causante de una alta morbilidad a nivel hospitalario. La clínica de la infección es amplia variando desde portadores asintomáticos hasta colitis fulminante, siendo la diarrea aguda la principal manifestación. El diagnóstico siempre se sospecha en cualquier paciente con diarrea aguda y factores de riesgo; y se confirma con una prueba en heces capaz de detectar las toxinas o el gen del Clostridium difficile. El tratamiento dependerá de la severidad de la infección, por lo general utilizando antibióticos de amplio espectro con sensibilidad por la bacteria como metronidazol, vancomicina o fidaxomicina. En algunas ocasiones la cirugía está indicada aunque no es lo más común. En el caso de aquellos pacientes con múltiples recurrencias el tratamiento con mayor evidencia de tasa de curación es el trasplante de microbiota fecal. La prevención con medidas de aislamiento y lavado de manos es de suma importancia para prevenir la transmisión entre pacientes a nivel hospitalario.

PALABRAS CLAVE: Clostridium difficile; infección nosocomial; microbiota; trasplante fecal; gastroenterología.

\section{ABSTRACT}

Clostridium difficile is an anaerobic, gram-positive bacterium capable of colonizing the colon and producing acute diarrhea. It is one of the most important nosocomial infections that causes 
high morbidity at the hospital level. The clinical manifestations are extensive ranging from asymptomatic carriers to fulminant colitis, with acute diarrhea being the main manifestation. Diagnosis is always suspected in any patient with acute diarrhea and risk factors; and is confirmed with a stool test capable of detecting toxins or the Clostridium difficile gene. Treatment will depend on the severity of the infection, usually using broad-spectrum antibiotics sensitive to the bacteria such as metronidazole, vancomycin or fidaxomicin. Sometimes surgery is indicated, although it is not the most common. For patients with multiple recurrences, treatment with the highest evidence of a cure rate is fecal microbiota transplantation. Prevention with isolation and hand washing measures is of paramount importance to prevent transmission between patients at the hospital level.

KEYWORDS: Clostridium difficile; nosocomial infection; microbiota; fecal transplant; gastroenterology.

${ }^{1}$ Médico general, graduado de la Universidad de Ciencias Médicas (UCIMED). Cód. MED16091. Correo: marioa0894@gmail.com

${ }^{2}$ Médica general, graduada de la Universidad de Ciencias Médicas (UCIMED). Cód. MED16050. Correo: bermudezlau96@outlook.es

${ }^{3}$ Médica general, graduada de la Universidad de Ciencias Médicas (UCIMED). Cód. MED16075. Correo: dianarojas2495@gmail.com

\section{INTRODUCCIÓN}

El Clostridium difficile es una bacteria anaeróbica, gram positiva, formadora de esporas; capaz de colonizar el intestino grueso y producir un amplio espectro de manifestaciones clínicas que van desde portadores asintomáticos hasta una colitis fulminante. Es hasta el año de 1978 que se describe por primera vez a la bacteria como la causante principal de colitis asociada a antibióticos (1). Esto llama la atención en una era donde el uso indiscriminado de antibióticos predispone a esta condición. El Clostridium difficile es uno de los principales causantes de infecciones nosocomiales asociado a una alta morbilidad y mortalidad con altos costos para el sistema de salud (2). El objetivo de esta revisión bibliográfica se enfoca en dar a conocer las características generales de la infección por Clostridium difficile, con el fin de reconocer esta patología y abordarla de la forma correcta para mejorar el pronóstico de los pacientes. También se explicará el papel del trasplante de microbiota fecal como una herramienta fundamental para combatir la infección.

\section{MÉTODO}

Para la elaboración de esta revisión bibliográfica, se revisaron diferentes artículos de los últimos 5 años en bases de datos como Uptodate, New England Journal of Medicine, Journal of association of medical microbiology and infectious disease of Canada y British Society of gastroenterology con el objetivo de tener una perspectiva amplia de varias entidades internacionales. Se utilizaron un total de 16 referencias bibliográficas.

\section{EPIDEMIOLOGÍA}

Está catalogada la infección por Clostridium difficile, como la causa más común de diarrea infecciosa en un contexto hospitalario. Con una incidencia anual en los EEUU de 142.7 casos por cada 100,000 personas (3). A partir del año 2000 ha habido un aumento significativo de los casos de infección nosocomial por Clostridium difficile y en el año 2011 este agente infeccioso se convirtió en el microorganismo más aislado en diferentes hospitales a nivel internacional $(2,3)$. 
El patrón epidemiológico de la infección por Clostridium difficile puede presentarse en la forma de 3 escenarios distintos: portador asintomático, infección nosocomial o infección adquirida en la comunidad. En el caso de portadores asintomáticos expuestos a un ambiente hospitalario, la incidencia puede variar según la literatura de un 3 a un $26 \%$, en contraste con tan solo menos del $2 \%$ en portadores asintomáticos sin exposición reciente a un ambiente hospitalario (3). La importancia del portador asintomático radica en que se comportan como reservorios, siendo capaces de esparcir esporas y contaminar a otros pacientes hospitalizados (1).

Factores como la edad y la respuesta inmune de la persona, son los que determinan el espectro de presentación de la enfermedad (4). Otro escenario clínico importante de la enfermedad es la infección recurrente, que se define como reaparición de los síntomas en un periodo de 2 a 8 semanas de haber completado el tratamiento. Esta presentación se llega a dar en hasta el $25 \%$ de todos los casos (1), por lo que representa un problema importante en el manejo para el clínico.

\section{PATOFISIOLOGÍA Y FACTORES DE RIESGO}

Para que llegue a suceder la infección por Clostridium difficile, primero debe de haber una colonización por parte de la bacteria en el colon. La forma en cómo se adquiere la bacteria es por la vía fecal-oral o por contacto con superficies contaminadas por esporas (3). Sin embargo, se necesitan de otros factores para que se establezca la infección, siendo uno de los más importantes la exposición a antibióticos. El uso de antibióticos produce una alteración del microbioma intestinal generando un ambiente óptimo para la colonización por Clostridium difficile, pues al disminuir las bacterias del microbioma intestinal se eliminan las defensas propias del huésped para impedir el crecimiento del Clostridium $(1,4)$. La disbiosis que se genera por el uso de antibióticos puede persistir hasta por 3 meses (3), por lo que en ese tiempo existe el riesgo de adquirir la infección.

La edad avanzada es otro de los factores predisponentes, pues son pacientes con una alta comorbilidad, por lo general se ven sometidos a un excesivo uso de antibióticos y cuentan con un mayor tiempo de hospitalización. También los agentes quimioterapéuticos, pues producen un efecto similar a los antibióticos en la microbiota intestinal. En el caso de los inhibidores de bomba de protones los estudios son controversiales, pues algunos creen que la disminución crónica de ácido a nivel gástrico predispone a una mayor entrada de bacterias, incluidas las esporas del Clostridium al tracto gastrointestinal $(2,3)$.

La bacteria no es enteroinvasiva, su mecanismo de acción es mediante la producción de dos exotoxinas, la toxina $A$ y B. Estas son capaces de inactivar las proteínas de la familia Rho, un componente importante del citoesqueleto, produciendo muerte de los colonocitos, pérdida de la barrera intestinal y colitis neutrofílica (1).

\section{MANIFESTACIONES CLÍNICAS}

Las manifestaciones clínicas de la infección por Clostridium difficile abarcan un amplio espectro que va desde una forma asintomática hasta una colitis fulminante. Lo más común es la diarrea acuosa (más de 3 deposiciones líquidas en 24 horas), asociado a datos de colitis (6). Según la severidad de la enfermedad, se puede clasificar en una enfermedad no severa caracterizada por diarrea, dolor abdominal tipo cólico, fiebre de bajo grado, náuseas y anorexia. Es típico una leucocitosis alrededor de 15000, con un examen físico 
caracterizado por sensibilidad abdominal baja (7).

La enfermedad severa se caracteriza por dolor abdominal difuso, distensión abdominal, fiebre mayor a $38,5^{\circ} \mathrm{C}$, hipovolemia, hipoalbuminemia, acidosis láctica. No existen criterios objetivos o una herramienta estandarizada para definir enfermedad severa de una no severa. Sin embargo, según la opinión de expertos una leucocitosis mayor a 15000 y una creatinina mayor a $1,5 \mathrm{mg} / \mathrm{dl}$ se considera enfermedad severa $(2,4)$

Otra presentación de la enfermedad aún más peligrosa es la colitis fulminante (anteriormente conocida como infección por Clostridium difficile complicada). Esta se caracteriza por cumplir con uno a más de los siguientes criterios: presencia de hipotensión o shock, presencia de un íleo, o datos radiográficos de un megacolon (6). Las manifestaciones de una colitis fulminante son bastante dramáticas, caracterizadas por una hipotensión que conlleva a falla multiorgánica, un íleo caracterizado por distensión abdominal severa, leucocitosis marcada y pocas deposiciones diarreicas, un megacolon que se determina por un diámetro del colon mayor a $7 \mathrm{~cm}$ en imágenes radiológicas (3, 5). La infección recurrente por Clostridium difficile es quizá una de las presentaciones que presenta el mayor reto para los clínicos. Se caracteriza por la reaparición de los síntomas 2 a 8 semanas después de haber completado un ciclo de tratamiento de forma exitosa (8). La gran mayoría de los episodios de recurrencia se deben a una recaída con la cepa inicial, y no una reinfección con una cepa diferente (6).

\section{DIAGNÓSTICO}

El diagnóstico de la infección por Clostridium difficile es relativamente fácil de realizar, siempre y cuando se tenga la sospecha clínica de la misma. Los diferentes exámenes de heces disponibles sólo se realizan en aquellos pacientes con heces líquidas (más de 3 deposiciones en 24 horas) y que tengan algún factor de riesgo (6).

El objetivo de los test es identificar el gen del Clostridium difficile o la presencia de las toxinas $A$ y $B$ en las heces. Los utilizados en la práctica clínica son el test de amplificación de ácido nucleico (NAAT), inmunoensayo enzimático para el antígeno glutamato deshidrogenasa (GDH) e inmunoensayo enzimático para las toxinas $A$ y $B$ (9). Cualquiera de los test se puede utilizar para hacer el diagnóstico. Las recomendaciones según diferentes guías es utilizar el NAAT como único test o en combinación con inmunoensayos. Se debe tener presente que un NAAT positivo no diferencia entre infección activa y portador asintomático, por lo que en ese caso se utiliza el inmunoensayo para las toxinas $A$ y $B$. Si el test inicial utilizado es un inmunoensayo, se recomienda combinarlo en un algoritmo de varios pasos incluyendo un NAAT $(2,7,9)$.

No hay evidencia que repetir el test aumente la sensibilidad, tampoco se recomienda realizar el test en personas asintomáticas con heces normales ya que los portadores asintomáticos no requieren tratamiento ni medidas de aislamiento $(6,9)$.

Los estudios de gabinete como radiografías de abdomen, TAC ayudan más a descartar enfermedad complicada. Si hay evidencia de una dilatación colónica de más de $7 \mathrm{~cm}$ de diámetro se sospecha de un megacolon tóxico, aire libre en cavidad peritoneal de una perforación intestinal. La endoscopia tiene como papel descartar otras causas de diarrea y colitis. La evidencia de una pseudomembrana es altamente sugestiva de infección por Clostridium difficile (6).

\section{TRATAMIENTO}

El tratamiento de la infección por Clostridium difficile se basa en antibióticos capaces de 
acabar con esta bacteria y sólo en determinados escenarios clínicos es necesario el manejo quirúrgico. El manejo médico varía según si es un episodio inicial, la severidad de este o si es más bien una presentación recurrente. La mayoría de las guías internacionales coinciden que como medidas generales se debe descontinuar en la medida de lo posible cualquier antibiótico incitante, con el objetivo de reducir el riesgo de recurrencia $(2,3)$. Además, se debe dar el manejo de posibles déficits hidroelectrolíticos secundarios a la diarrea, una dieta libre de lactosa no es necesaria y no hay evidencia suficiente para recomendar la suspensión de cualquier agente antimotilidad como la loperamida (8).

Muchas guías internacionales coinciden en dar un manejo médico dependiendo si es un episodio inicial o una recurrencia; y en el caso de un episodio inicial dependerá de la gravedad del mismo ya sea enfermedad no severa (leve, moderada), enfermedad severa, colitis fulminante o complicada. Para el episodio inicial no severo se recomienda vancomicina oral $125 \mathrm{mg}$ QID por 10 días o fidaxomicina $200 \mathrm{mg}$ vía oral dos veces al día por 10 días $(3,4)$. El metronidazol es una alternativa como primera línea, pero actualmente se sabe que el uso de este antibiótico aumenta la posibilidad de recurrencia, sobre todo en personas mayores de 65 años (8). El tratamiento recomendado para la enfermedad severa es vancomicina o fidaxomicina con las mismas dosis anteriormente descritas. metronidazol no tiene papel es este escenario clínico. El uso de fidaxomicina demuestra una tasa de recurrencia menor en comparación con vancomicina (7).

En el caso de la enfermedad complicada o colitis fulminante se recomienda vancomicina oral con una dosis que puede variar desde $125 \mathrm{mg}$ a $500 \mathrm{mg} 4$ veces al día en conjunto con metronidazol intravenoso $500 \mathrm{mg}$ cada 8 horas (2,3). Además, se debe considerar en el caso de un íleo la vía rectal en forma de enema para la administración de vancomicina (3).La enfermedad recurrente por Clostridium difficile conlleva un reto para los clínicos, pues la probabilidad de recurrencia es alta y una vez que ocurra un episodio de recurrencia, mayor la posibilidad de otros episodios (1).

El manejo para una primera recurrencia es el mismo que el episodio inicial. Sin embargo, se recomienda vancomicina o fidaxomicina en caso de haber utilizado metronidazol en el primer episodio (2). En el caso de una segunda recurrencia se recomienda utilizar vancomicina oral en un régimen en pulsos con una reducción paulatina de la dosis a lo largo de varias semanas, otra opción es agregar rifaximina después del régimen de vancomicina $(7,8)$.A partir de una tercera recurrencia o cuando el paciente falla a al menos un ciclo con reducción paulatina de vancomicina, se recomienda el uso del trasplante de microbiota fecal $(2,3,8)$.

Se debe solicitar consulta a cirugía general en caso de sospecha de enfermedad complicada, como fiebre más de $38,5^{\circ} \mathrm{C}$, hipotensión, datos de peritonitis, íleo o distensión abdominal importante, lactato sérico mayor a $2,2 \mathrm{mmol} / \mathrm{L}$, leucocitosis mayor a 20000 (8).

Se han hecho estudios sobre nuevas terapias para combatir la infección, uno de ellos es el bezlotoxumab. El mecanismo de acción de este consiste en brindar una inmunidad pasiva contra la toxina $B$ del Clostridium difficile al bloquear su unión con las células de la persona. La indicación de este medicamento tiene mayor evidencia en aquellas personas en riesgo de presentar una recurrencia después de un primer ciclo de antibiótico (10), por lo que se puede utilizar como una alternativa de tratamiento.

\section{PAPEL DEL TRASPLANTE DE MICROBIOTA FECAL}

El uso de trasplante de microbiota fecal (TMF) se basa en el hecho de que la 
microbiota colónica diversa protege frente a la colonización por el Clostridium difficile (2). Pacientes con episodios recurrentes de infección han demostrado tener una reducción en las especies de bacterias de su microbioma, por lo que el trasplante fecal de individuos sanos rompe el ciclo de recurrencia (11). La mayoría de los estudios y ensayos clínicos en el uso del trasplante de microbiota fecal se basan en episodios de enfermedad recurrente. De ahí que la indicación de este tratamiento debe ser sólo en caso de dos o más recurrencias por Clostridium difficile (12). Otras guías recomiendan la posibilidad de utilizar el TMF en pacientes con enfermedad severa o complicada que no responden a las 48 horas de terapia convencional (5). A raíz de la poca evidencia que existe en el uso de TMF en episodios iniciales, no se recomienda su uso como manejo inicial. También existen contraindicaciones de su uso, por el riesgo de trasmisión de enfermedades infectocontagiosas, por lo que está contraindicado en pacientes inmunosupresos y pacientes con neutropenia severa $(11,12)$. La eficacia del trasplante de microbiota fecal es bastante alta, pues según estudios que compararon su uso con un régimen estándar de vancomicina oral, demostraron una curación del $81 \%$ en el caso de TMF con tan solo un $31 \%$, con lo que se demostró una eficacia mucho mayor para curación en pacientes con enfermedad recurrente (13). Muchos estudios randomizados también apoyan estos datos, pues demuestran una tasa de curación en enfermedad recurrente de entre 70 a $90 \%$ en un seguimiento de hasta 18 meses (8).Se debe seguir un procedimiento para llevar a cabo el TMF, incluyendo la recolección de las heces de un donante, el tamizaje de las heces por enfermedades infecciosas, la preparación de la muestra y la ruta de administración. En el caso del donante se recomienda que sea una persona entre 18 y 60 años, de peso adecuado y que se les realice una historia clínica en busca de enfermedades infecciosas $o$ factores que alteran la microbiota fecal como uso de antibióticos en los últimos 3 meses, riesgo de enfermedades de trasmisión sexual, historia de enfermedades gastrointestinales como SII o Ell (14). Las heces que se utilizaran se deben testear por parásitos, Clostridium y bacterias multirresistentes. En el donante se hacen exámenes serológicos como hepatitis virales, VIH, Ebsien Var y citomegalovirus (5, 14). Para la preparación de las heces no hay una guía específica a seguir. Sin embargo, se recomienda utilizar más de $50 \mathrm{~g}$ de heces, diluir con SF 0,9\% alrededor de 100 a $700 \mathrm{ml}$ y mezclarlo hasta obtener una mezcla homogénea (5). La ruta de administración puede ser por tracto gastrointestinal alto por medio de una sonda nasoduodenal o nasoyeyunal, también por medio de cápsulas orales; o por la vía gastrointestinal baja ya sea mediante una colonoscopia o por la forma de enema (7).

La eficacia según las diferentes rutas de administración es incierta. Sin embargo, se sabe que las técnicas que utilizan el tracto gastrointestinal bajo tienen una mayor eficacia en comparación con vía alta, también se ha demostrado que al dar más de una infusión de microbiota fecal aumenta la eficacia de curación por encima de un $80 \%$ $(2,11)$. A pesar de esto, la recomendación general es utilizar la ruta más adecuada según las características de cada paciente. Por ejemplo, en el caso de la existencia de una colectomía parcial o colostomía se prefiere la vía alta o en el caso de un paciente con una lesión obstructiva a nivel esofágico se optaría por la vía baja (12).

\section{PROTOCOLO DE ADMINISTRACIÓN DEL TRASPLANTE DE MICROBIOTA FECAL}

Una vez que se cuente con la muestra de microbiota fecal lista para su administración 
y la vía adecuada de infusión para el paciente se deben de seguir una serie de pasos para lograr un trasplante de microbiota adecuado. Los pasos varían según la ruta de administración, pero los diferentes estudios concuerdan en que hay un beneficio en dar un régimen antibiótico, independientemente de la ruta, días antes del procedimiento y que la última dosis debe de darse como máximo 24 horas antes del mismo (11). No hay una guía exacta de cuál antibiótico o dosis es la adecuada (7). También existe un consenso en que se debe preparar el colon con una solución de polietilenglicol el día antes del procedimiento para cualquier vía de administración, con el objetivo de reducir la carga bacteriana en el colon. En el caso de utilizar la vía gastrointestinal alta, se debe dar la noche antes del procedimiento un inhibidor de bomba de protones y el día del mismo se recomienda administrar un proquinético 30 minutos antes del procedimiento. Al utilizar la vía baja se deben administrar entre 200 a $300 \mathrm{~g}$ de heces disueltos en 200 a $300 \mathrm{ml}$ de solución y para la vía alta se recomienda no administrar más de $100 \mathrm{ml}(5,11,12)$.

Posterior al trasplante de microbiota fecal se debe vigilar por efectos adversos como fiebre, dolor abdominal y vómitos. Por lo general el paciente debería de tener mejoría clínica a los 2 o 3 días desde la aplicación del procedimiento. Se considera exitoso el mismo si no hay recurrencia de los síntomas a los 2-3 meses, si hubiese una recurrencia antes de ese tiempo estaría indicado repetir el trasplante $(5,11)$.

\section{PREVENCIÓN DE LA INFECCIÓN POR Clostridium difficile}

Dada la alta morbilidad y mortalidad que representa la infección por Clostridium difficile a nivel hospitalario, es importante la implementación de medidas para prevenir la trasmisión de esta enfermedad tanto a los pacientes como al personal sanitario. Se recomienda una vez diagnostica la infección establecer medidas de precaución de contacto, como colocar al paciente en una habitación privada, colocar guantes y batas en la entrada de la habitación las cuales se deben descartar cada vez que se entra en la misma, realizar lavado de manos después de tener contacto con el paciente y entre pacientes. Se recomienda que la duración del aislamiento sea hasta 48 después de la resolución de la diarrea $(15,16)$. Está demostrado que el uso de soluciones con alcohol no elimina las esporas de Clostridium difficile, por lo que se recomienda el lavado de manos con agua y jabón en su lugar (4).

La limpieza de las superficies hospitalarias, sobre todo aquellas que tienen más contacto con el paciente como la cama, la mesita de noche, los pasamanos son capaces de mantener las esporas por meses. Por esta razón se recomienda realizar una limpieza de estas superficies con un desinfectante todos los días y en el caso de un brote de Clostridium se recomienda utilizar un agente esporicida para la limpieza (16).

Se cree que los individuos portadores asintomáticos son capaces de transmitir la infección al ambiente, aunque en menor medida que las personas sintomáticas. En el caso de descubrir un paciente portador asintomático, no hay indicación clara de qué medidas se deben tomar para prevenir la transmisión, por lo que no se puede dar una recomendación (6).

\section{CONCLUSIONES}

La infección por Clostridium difficile es una de las infecciones nosocomiales con mayor morbilidad y mortalidad en cualquier región del mundo. De aquí la importancia de tener los conocimientos clínicos adecuados para realizar un diagnóstico oportuno y dar un manejo adecuado de la misma. Por tal razón se debe considerar siempre el diagnóstico por Clostridium en aquellos pacientes con una diarrea aguda, sobre todo con factores 
de riesgo tanto a nivel hospitalario como en la comunidad.

Un problema importante de esta patología es su porcentaje alto de recurrencia y el fallo al tratamiento con la terapia antibiótica convencional, por tal razón el uso de terapias innovadoras como es el trasplante de microbiota fecal la convierte en la mejor opción actual, pues cuenta con la mayor evidencia científica a nivel internacional en cuanto a tasa de curación.

Es importante resaltar que el personal médico debe comprender la base fisiopatológica de la enfermedad, donde el uso innecesario de antibióticos de amplio espectro predispone a la colonización del colon por esta bacteria. De ahí la importancia de hacer un uso adecuado de los mismos para prevenir la infección por Clostridium difficile, principalmente en personas en riesgo y de esta forma disminuir su impacto en el sistema de salud. Se debe hacer un esfuerzo en implementar protocolos a nivel hospitalario en el manejo de pacientes con Clostridium difficile con el objetivo de prevenir la transmisión al resto de pacientes internados, estos protocolos se deben basar en un adecuado uso de medidas de aislamiento de contacto y en un adecuado programa de lavado de manos.

Una vez que el clínico comprenda lo anteriormente descrito será capaz de dar un manejo de esta patología y aún más importante una prevención de la forma más adecuada, con las bases científicas más recientes y en beneficio del paciente.

\section{Los autores declaran no tener conflicto de interés.}

\section{REFERENCIAS}

1. Lamont J, Kelly C, Bakken J. Clostridioides (formerly Clostridium) difficile infection in adults: Epidemiology, microbiology, and pathophysiology. Uptodate. Wolters Kluwer [internet]. 4 agosto 2020 [citado 10 marzo 2021]; [aprox. 28 p.]. Disponible en:

https://www.uptodate.com/contents/clostridioides- formerly-clostridium-difficile-infection-in-adultsepidemiology-microbiology-and-pathophysiology

2. Loo V, Davis I, Embil J, Evans G, Hota S, Lee S et al. Association of Medical Microbiology and Infectious Disease Canada treatment practice guidelines for Clostridium difficile infection. Official $\mathrm{J}$ AssocMed Microbiol Infect Dis Canada. [internet]. 2018 [citado 10 de marzo 2021]; 3(2): 71-92. https://doi.org/10.3138/jammi.2018.02.13

3. McDonald LC, Gerding D, Johnson S, Bakken J, Carrol K, Coffin S, et al. Clinical Practice Guidelines for Clostridium difficile Infection in Adults and Children: 2017 Update by the Infectious Diseases Society of America (IDSA) and Society for Healthcare Epidemiology of America (SHEA). Clin Infect Dis. 2018; 66(7): e1e48. https://doi.org/10.1093/cid/cix1085

4. Kelly C, Lamont J. Antibiotic associated diarrhea and Clostridium difficile infection. En: Mark Feldman, Lawrence S. Friedman, Lawrence J. Brandt. Sleisenger and Fordtran. Gastrointestinal and liver disease. 10th ed. Philadelphia: Elsevier; 2016. p 1939-1954

5. Davidovics Z, Michail S, Nicholson M, Kociolek L, Pai $N$, Hansen $R$, et al. Fecal Microbiota Transplantation for Recurrent Clostridium difficile Infection and Other Conditions in Children: A Joint Position Paper From the North American Society for Pediatric Gastroenterology, Hepatology, and Nutrition and the European Society for Pediatric Gastroenterology, Hepatology, and Nutrition. J Pediatr Gastroenterol Nutr. [internet]. 2019 [citado 10 marzo 2021]; 68(1): 130-143 https://doi.org/10.1097/MPG.0000000000002205

6. Lamont J, Kelly C, Bakken J. Clostridioides (formerly Clostridium) difficile infection in adults: Clinical manifestations and diagnosis. Uptodate. Wolters Kluwer [internet]. 9 diciembre 2020 [citado 10 marzo 2021]; [aprox. 33 p.]. Disponible en: https://www.uptodate.com/contents/clostridioidesformerly-clostridium-difficile-infection-in-adultsclinical-manifestations-and-diagnosis

7. Bagdasarian N, Rao K, Malani PN. Diagnosis and treatment of Clostridium difficile in adults: a systematic review. JAMA. 2015; 313(4):398-408. doi: 10.1001/jama.2014.17103. PMID: 25626036; PMCID: PMC6561347.

8. Kelly C, Lamont J, Bakken J. Clostridioides (formerly Clostridium) difficile infection in adults: Treatment and prevention. Uptodate. Wolters Kluwer [internet]. 24 febrero 2021 [citado 10 marzo 2021]; [aprox. 36 p.]. Disponible en: https://www.uptodate.com/contents/clostridioidesformerly-clostridium-difficile-infection-in-adultstreatment-and-prevention

9. Caliendo A. Lab Interpretation: Positive Clostridioides (formerly Clostridium) difficile in adults. Uptodate. Wolters Kluwer [internet]. 5 
mayo 2020 [citado 10 marzo 2021]; [aprox. 6 p.].

Disponible

en:

https://www.uptodate.com/contents/positive-

clostridioides-formerly-clostridium-difficile-in-

adults

10. Bartlett J. Bezlotozumad - A new agent for Clostridium difficile infection. $\mathrm{N}$ engl $\mathrm{J}$ med [internet]. 2017 [citado 10 marzo 2021]; 376(4): 381-382. https://doi.org/10.1056/NEJMe1614726

11. Borody $\mathrm{T}$, Ramrakha $\mathrm{S}$. Fecal microbiota transplantation for treatment of Clostridioides (formerly Clostridium) difficile infection. Uptodate. Wolters Kluwer [internet]. 1 febrero 2021 [citado 10 marzo 2021]; [aprox. 20 p.]. Disponible en: https://www.uptodate.com/contents/fecalmicrobiota-transplantation-for-treatment-ofclostridioides-formerly-clostridium-difficileinfection

12. Mullish BH, Quraishi MN, Segal JP, et al. The use of faecal microbiota transplant as treatment for recurrent or refractory Clostridium difficile infection and other potential indications: joint British Society of Gastroenterology (BSG) and Healthcare Infection Society (HIS) guidelines. Gut. 2018; 67(11): 1920-1941. https://doi.org/10.1136/gutjnl2018-316818

13. Nood E, Vrieze A, Nieuwdorp M, Fuentes S, Zoetendal E, de Vos W, et al. Duodenal Infusion of Donor Feces for Recurrent Clostridium difficile. $\mathrm{N}$ engl J med [internet]. 2016 [citado 10 marzo 2021]; 368(5): 407-415. https://doi.org/10.1056/NEJMoa1205037

14. Kassam Z, Dubois N, Qazi T, Smith M, Kelly C, Fischer $M$, et al. Donor Screening for Fecal Microbiota Transplantation. $\mathrm{N}$ engl J med [internet]. 2019 [citado 10 marzo 2021]; 381(21):[aprox. 3 p.]. https://doi.org/10.1056/NEJMc1913670

15. Doll M, Marra A, Apisarnthanarak A, Saif Al-Maani A, Abbas S, Rosenthal V. Prevention of Clostridioides difficile in hospitals: A position paper of the International Society for Infectious Diseases. Int J Infect Dis [internet]. 2021 [citado 10 marzo 2021]; 102: 188-195. https://doi.org/10.1016/j.ijid.2020.10.039

16. Clifford L, Kutty P. Clostridioides (formerly Clostridium) difficile infection: Prevention and control. Uptodate. Wolters Kluwer [internet]. 24 febrero 2019 [citado 10 marzo 2021]; [aprox. 19 p.]. Disponible en: https://www.uptodate.com/contents/clostridioidesformerly-clostridium-difficile-infection-preventionand-control 